\title{
Low content of the natural ocular anti-angiogenic agent pigment epithelium-derived factor (PEDF) in aqueous humor predicts progression of diabetic retinopathy
}

\author{
B. O. Boehm ${ }^{1}$, G. Lang ${ }^{2}$, O. Volpert ${ }^{3,4}$, P. M. Jehle ${ }^{1}$, A. Kurkhaus ${ }^{1}$, S. Rosinger ${ }^{1}$, G. K. Lang ${ }^{2}$, N. Bouck ${ }^{4,5}$ \\ ${ }^{1}$ Division of Endocrinology and Diabetes, Department of Internal Medicine, University of Ulm Medical School, Ulm, Germany \\ 2 Department of Ophthalmology, University of Ulm Medical School, Ulm, Germany \\ ${ }^{3}$ Department of Urology, Northwestern University Medical School, Chicago, USA \\ ${ }^{4}$ The Robert H. Lurie Comprehensive Cancer Center, Northwestern University Medical School, Chicago, USA \\ ${ }^{5}$ Department of Microbiology-Immunology, Northwestern University Medical School, Chicago, USA
}

\begin{abstract}
Aims/hypothesis. Retinopathy is the most common microvascular complication of diabetes. Our aim was to address the predictive value of pro-angiogenic and anti-angiogenic markers for progression of retinopathy. Methods. Aqueous humor was collected at cataract surgery from 32 diabetic patients who had no or very mild retinopathy (ETDRS stage $\leq 20$ ) and 33 normoglycaemic control subjects. Content of pro-angiogenic vascular endothelial growth factor and angiogenic inhibitor pigment epithelium-derived factor were determined. Angiogenic activity was quantified by measuring its effect on the migration of capillary endothelial cells. The predictive value of the initial level of these markers for progression of retinopathy was studied by following the probands for a maximum of 75 months. Results. In the aqueous fluid content of vascular endothelial growth factor was increased in diabetic patients (mean values 492 versus $292 \mathrm{pg} / \mathrm{ml} ; p=0.0052$ ), and pigment epithelium-derived factor values were decreased (mean values 1740 versus $3680 \mathrm{ng} / \mathrm{ml}$; $p=0.0058$ ) compared to control subjects. Of the diabetic patients ten progressed during follow-up (ETDRS
\end{abstract}

stage $>47 \mathrm{~B}$ ). This subgroup showed lower pigment epithelium-derived factor content when compared to non-progressors and control subjects. Migratory activity in samples of patients from the control group and in diabetic patients without progression was generally inhibitory due to pigment epithelium-derived factor. Inhibition was blocked by neutralizing antibodies to pigment epithelium-derived factor. In diabetic patients initial angiogenic activity was higher in those who later developed retinopathy (vs. controls $p=0.00005$; vs. no progressors $p=0.0003$ ). Both pigment epitheliumderived factor and migratory response predicted progression.

Conclusion/Interpretation. Pigment epithelium-derived factor is an important negative regulator of angiogenic activity of aqueous humor. Its content in the aqueous humor of diabetic patients strongly predicts who among them will develop progression of retinopathy. [Diabetologia (2003) 46:394-400]

Keywords Angiogenesis, angiogenic inhibitors, aqueous humor, diabetic retinopathy, pigment epitheliumderived factor (PEDF), vascular endothelial growth factor (VEGF).
Received: 23 August 2002 / Revised: 10 October 2002

Published online: 1 March 2003

(C) Springer-Verlag 2003

Corresponding author: B. O. Boehm MD, Division of Endocrinology and Diabetes, Department of Internal Medicine, University of Ulm Medical School, Robert-Koch-Strasse 8, 89081 Ulm, Germany

E-mail: bernhard.boehm@medizin.uni-ulm.de

Abbreviations: EBM, endothelial cell basal medium; ETDRS, early treatment of diabetic retinopathy study; PEDF, pigment epithelium-derived factor; VEGF, vascular endothelial growth factor.
Retinopathy is the most frequent chronic microvascular complication associated with diabetes mellitus. It represents a major threat to eyesight in Western countries, and is still the leading cause of blindness among people of working age [1]. Although intensive glycaemic control can delay the onset of diabetic retinopathy and ameliorate the severity of the disease, both in Type 1 and Type 2 diabetic patients [2, 3, 4], the incidence of diabetic retinopathy remains startlingly high $[1,5]$. It eventually affects the majority of diabetics, progressing to legal blindness in about $5 \%$ of them $[1,5,6]$. 
Progressive diabetic retinopathy involves an angiogenic switch where normally quiescent vasculature begins to grow. Mild diabetic retinopathy does not pose an immediate threat to vision and can remain stable over many years. However, it constitutes a serious risk since it is an essential precursor to proliferative retinopathy, in which invasive vessels can compromise vision by growing into the ischaemic portions of the retina to cause detachment and/or entering the avascular vitreous to haemorrhage $[1,6,7]$.

Despite intensive study of retinopathy in recent years, few have examined patients prior to the development of abnormal vessels or have followed such individuals prospectively. Much attention has been paid to the mechanisms of neovascularization in the later stages of retinopathy. Diabetic retinopathy, which is probably ischaemia driven, is accompanied by an increase in a number of angiogenic growth factors including hypoxia-induced vascular endothelial growth factor (VEGF) [7, 8, 9, 10, 11]. VEGF, which increases microvascular permeability and acts as an endothelial-cell selective mitogen, is considered a key factor in the disease $[7,9,10]$. Its content in the vitreous correlates with severity of the disease and antagonists of VEGF or its specific receptor ameliorate retinopathy in animal models [12].

However, the switch from quiescent to active vessels often involves not only an increase in inducers of neovascularization but also a decrease in the concentration of negative regulators of angiogenesis, thus resulting in the breaking of the delicate balance between angiogenic stimulators and inhibitors [13, 14]. One such natural inhibitor in the eye is pigment epithelium-derived factor $[15,16]$. It is responsible for both the anti-angiogenic activity of human vitreous and for excluding vessels from the cornea $[15,16]$. Regulated by oxygen concentrations, PEDF behaves in a manner opposite to stimulatory VEGF, falling in concentration when oxygen is limited and rising when it is in good supply $[15,17,18,19]$. Recent data have provided evidence that PEDF is not only produced by the pigment epithelium but can also be found at high concentrations in most compartments of the eye, including the anterior chamber [20]. PEDF shows a dual function, firstly being antiangiogenic and secondly acting as a neutrophic factor promoting the survival of a variety of neuronal cells including photoreceptors $[15,16,21$, 22, 23].

To address the role of pro-angiogenic and anti-angiogenic factors in the early stages of diabetic retinopathy, we have determined aqueous humor content of the natural angiogenic inhibitor PEDF and of the angiogenesis stimulator VEGF. Also migratory activity in aqueous humor samples was obtained to address the functional role of pro-angiogenic and anti-angiogenic factors.

\section{Subjects and Methods}

Patients. Patients were recruited from the Department of Ophthalmology of the University Hospital in Ulm. Diabetic patients with cataract and non-diabetic patients with cataract only (controls) were recruited from January 1995 to December 1995, and followed until December 2001. Mean follow-up was 69 months (range: 64-75 months). Inclusion criteria were the presence of lense opacities that had to be treated by cataract extraction and lack of evidence for mild to severe non-proliferative retinopathy, i.e. ETDRS scale less than or equal to 20. Exclusion criteria included, uncontrolled hypertension (systolic values $>170 \mathrm{mmHg}$, diastolic $\mathrm{RR}$ values $>95 \mathrm{mmHg}$ ), ETDRS scale greater than 20 at baseline and uncontrolled diabetes $\left(\mathrm{HbA}_{1 \mathrm{c}}\right.$ values $\left.>9 \%\right)$, to rule out probands that could show a so-called rebound phenomenon with an early worsening of diabetic retinopathy associated with lowering of blood glucose concentration $[2,3,6,24,25,26]$, macular oedema, including clinically significant macular oedema requiring grid laser photocoagulation. All patients signed written informed consent for their participation in the study. The study design was approved by the Ulm ethical committee.

Ophthalmologic follow-up. The patients were examined immediately after cataract extraction and aqueous humor collection. Examinations were repeated every 12 months during the follow-up period. The standard examination at every visit included measurement of visual acuity and measurement of intraocular pressure, examination of the anterior segment of the eye by slit-lamp biomicroscopy, gonioscopy to detect iris neovascularization, dilation of the pupil, direct and indirect ophthalmoscopy. Stereoscopic colour fundus photographs were taken every 12 months and were graded by observers masked to treatment and disease state. All members of the independent reading centre had been certified by the Fundus Photograph Reading Centre of the University of Wisconsin, Madison, USA. Grading of retinopathy was done according to the ETDRS scale. This scale divides diabetic retinopathy into over $20 \mathrm{lev}-$ els ranging from absence of retinopathy (scale of 10) to severe vitreous haemorrhage in advanced proliferative retinopathy (score over 81). When indicated, laser photocoagulation was used according to standard recommendations $[1,6]$.

Collection of aqueous humor. At the onset of cataract surgery aqueous fluid from the anterior chamber of the eye was obtained. A maximum of $200 \mu \mathrm{l}$ (range: 50-200 $\mu \mathrm{l}$ ) of undiluted fluid was drawn under sterile conditions. The fluid was immediately frozen and stored at $-80^{\circ} \mathrm{C}$ until assayed.

HbAlc assay. $\mathrm{HbA}_{1 \mathrm{c}}$ was determined using the principles of ion exchange HPLC (BioRad, Munich, Germany; normal range: $4.3-6.1 \%$ ).

VEGF assay. $\mathrm{VEGF}_{165}$ was quantified using a commercially available sandwich enzyme immunoassay technique (R\&D Systems, Wiesbaden-Nordenstadt, Germany).

Quantification of PEDF. Quantification of PEDF in clinical samples was carried out using immunoblots. To avoid loading errors samples were diluted 1 to 5 with saline and $5 \mu$ of the diluted sample was loaded resulting in $1 \mu \mathrm{l}$ of undiluted aqueous humor. Samples were run on at least two independent reducing SDS polyacrylamide gels along with serial dilutions of purified PEDF [15], transferred to PVDF membranes, blotted, probed with anti-PEDF affinity purified antibody [15] or probed with a monoclonal anti-PEDF antibody (Chemicon Int., 
Temecula, Calif., USA), and developed with a highly sensitive chemiluminescent kit (ECL kit, Amersham, Arlington Heights, Ill., USA). The blots were exposed to X-ray film, digital images obtained and quantified using ImageQuant densitometer (Molecular Dynamics, San Francisco, Calif., USA) and software, and PEDF concentration in the aqueous humor sample was calculated in relationship to the linear area of a standard curve generated using purified PEDF. Samples measured had been stored at $-80^{\circ} \mathrm{C}$ for almost $4-5$ years. Quality controls revealed no decay of PEDF values due to storage conditions used. Intra-assay and inter-assay precision less than 7\%, respectively.

Boyden test: In vitro angiogenesis assay. Endothelial cell migration assays were carried out $[15,27]$ and samples were diluted 1 to 10 in serum-free endothelial cell basal medium (EBM) containing $0.1 \%$ bovine serum albumin, and added to the top side of gelatinized microporous membranes $(8 \mu \mathrm{mol}$ pore size, Nucleopore, Plesanton, Calif., USA) in a modified Boyden chamber. Angiogenic activity was reflected by the ability of the samples to induce chemotaxis of neonatal human dermal microvascular endothelial cells (HMVECs from Clonetics Cell Systems, San Diego, Calif., USA), that had been plated at $10^{6}$ cells per $\mathrm{ml}$ on the opposite side of the membranes. As endothelial cells lose their tissue-specific qualities upon culture and since PEDF can affect endothelial cells in the eye and control angiogenesis in the cornea, the system used reflects migratory response. Each sample was tested in quadruplicate. EBM with bovine serum albumin served as a negative control (BSA) and the same medium containing vascular endothelial cell growth factor (VEGF) at $200 \mathrm{pg} / \mathrm{ml}$, as a positive control. Where indicated antibodies that had been affinity purified against a synthetic peptide and which do neutralize the anti-angiogenic activity of PEDF [15] were added at $20 \mu \mathrm{g} / \mathrm{ml}$. The antibodies used had no direct effect on angiogenesis. Intra-assay and inter-assay varation was less than 7\%, respectively.

Statistical analysis. Statistical calculations were done using the SAS package 6.12 (SAS Institute, Cary, N.C., USA). The Chi-square test was used for the analysis of categorial data. Student's $t$ test was used for the comparison of VEGF-, PEDF-, and $\mathrm{HbA}_{1 \mathrm{c}}$ values. A logistic regression model was used to address and to verify the association between the markers determined at baseline as a single explanatory variable for progression of retinopathy. In the model other explanatory variables including age of the probands, $\mathrm{HbA}_{1 \mathrm{c}}$ values, diabetes duration and hypertension were also taken into account. A $p$ value of less than 0.05 was considered statistically significant.

\section{Results}

Baseline characteristics. In 19951278 patients had cataract surgery, among them 358 were diabetic patients $(28 \%)$. Out of 358 diabetic patients 94 met inclusion criteria, 32 diabetics and 33 control subjects were recruited for obtaining aqueous fluid and for subsequent ophthalmological follow up (Table 1). As expected, the $\mathrm{HbA}_{1 \mathrm{c}}$ values were higher in the group of diabetic patients. Sex, age, and cigarette smoking habits were comparable between the diabetic and the control cohort. The group of diabetic patients mostly comprised of Type 2 diabetic patients. All diabetic
Table 1. Characteristics of the patients at enrolment

\begin{tabular}{llll}
\hline Characteristics & $\begin{array}{l}\text { Control } \\
\text { subjects } \\
(n=33)\end{array}$ & $\begin{array}{l}\text { Diabetic } \\
\text { patients } \\
(n=32)\end{array}$ & $p$ value \\
\hline Sex & & & \\
Male & 14 & 14 & n.s. \\
Female & 19 & 18 & n.s. \\
Age $(y r)$ & & & \\
Mean & 71.7 & 72.2 & n.s. \\
Range & $54-85$ & $55-90$ & \\
HbA 1 (\%) & & & \\
Mean & 5.56 & 7.19 & $0.001 * *$ \\
Range & $5.0-7.3$ & $5.1-9.0$ & \\
Type 1 Diabetics & n. a. & 4 & \\
Type 2 Diabetics & n. a. & 28 & \\
Insulin-treatment & n.a. & 13 & \\
OAD & n.a. & 19 & \\
Presence of Hypertension & 14 & 21 & $0.071^{*}$ \\
Cigarette smoking & 7 & 7 & n.s. \\
\hline
\end{tabular}

\# two non-diabetic probands showed a $\mathrm{HbA}_{1 \mathrm{c}}$ levels above the range of normal values $(6.1 \%)$ with 6.6 , and 7.3 , respectively. However, glucose levels were found to be in the normal range. Hypertension was defined by a RR value equal to or above 140/90 $\mathrm{mmHg}$ and/or use of anti-hypertensive medication.

\#\# Wilcoxon rank-sum test for the comparison of continuous data.

* Chi-square test for categorial parameters.

n.s. = not significant

n.a. $=$ not applicable

$\mathrm{OAD}=$ oral antidiabetic drug

probands had a good metabolic control with a mean $\mathrm{HbA}_{1 \mathrm{c}}$ of $7.19 \%$ (normal: $\leq 6.1 \%$ ). In the control group (mean $\mathrm{HbA}_{1 \mathrm{c}} 5.56 \%$ ) two subjects had a $\mathrm{HbA}_{1 \mathrm{c}}$ value above $6.1 \%$, which is above the upper limit of normal. However, repeated blood glucose measurements showed normal blood glucose values in these subjects. Hypertension (as defined by a blood pressure of $\geq 140 / 90 \mathrm{mmHg}$ and/or use of anti-hypertensive drugs) was found in $42 \%$ (14/33) of the control subjects but it was higher in the diabetic cohort [66\% (21/32); $p=0.071]$. At the end of the study period no remarkable change of $\mathrm{HbA}_{1 \mathrm{c}}$ values compared to baseline was found.

$V E G F, P E D F$, and $H b A_{1 c}$ values. Baseline VEGF content in the aqueous humor were increased in the cohort of diabetic patients $(p=0.0052)$ with a mean of $493 \mathrm{pg} / \mathrm{ml}$ (range: 57-2000) as compared to the control group [mean value $286 \mathrm{pg} / \mathrm{ml}$ (range: 50-608)]. PEDF values tended to be lower in the diabetic cohort with a mean value of $1488 \mathrm{ng} / \mathrm{ml}$ (range: 10-5300) compared to $3890 \mathrm{ng} / \mathrm{ml}$ (range: $10-1970 ; p=0.0058$ ) in the control group. The $\mathrm{HbA}_{1 \mathrm{c}}$ values showed no correlation to either VEGF or PEDF (Fig. 1, Fig. 2a). 


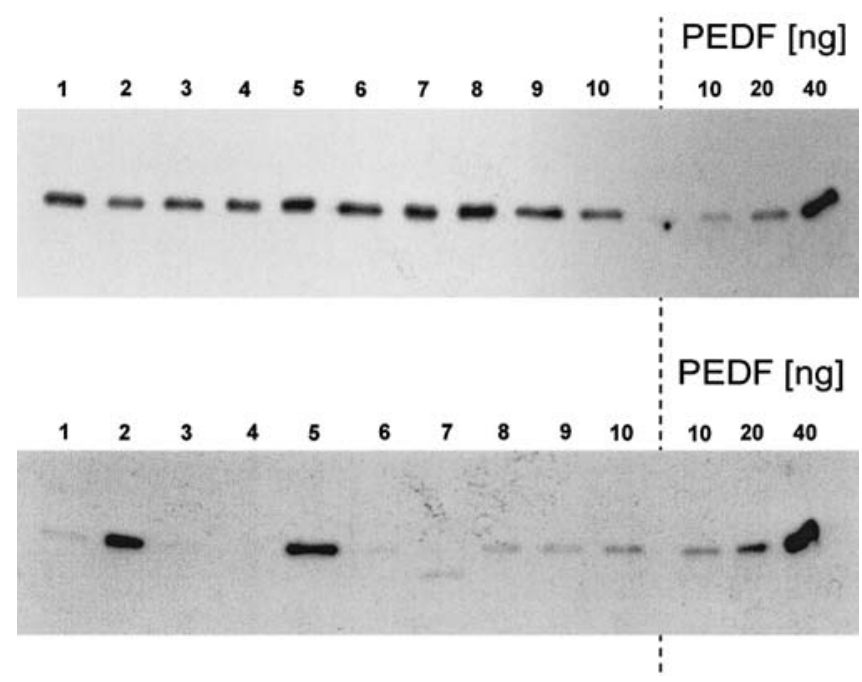

Fig. 1. PEDF determination by use of Western blot analysis. In the upper part samples of aqueous humor are shown from control subjects, lanes 1-10; on the right side corresponding PEDF standards are given. In the lower part lanes $1,3,4,6-10$ correspond to samples from diabetic patients who showed progression, whereas in lanes 2 and 5 aqueous humor samples from non-progressors are shown
A

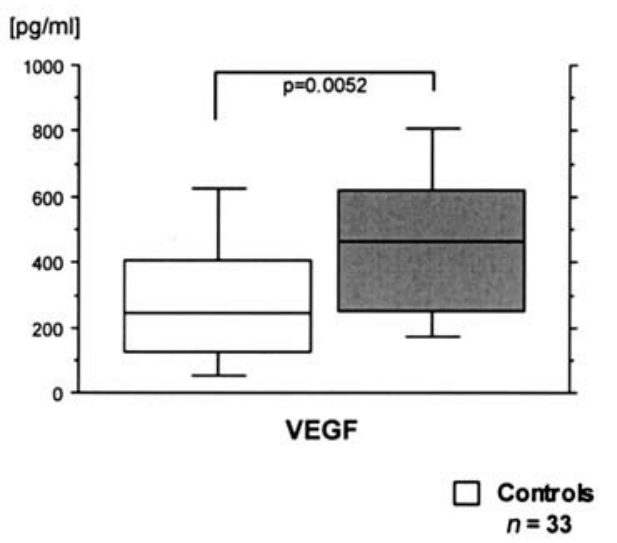

B

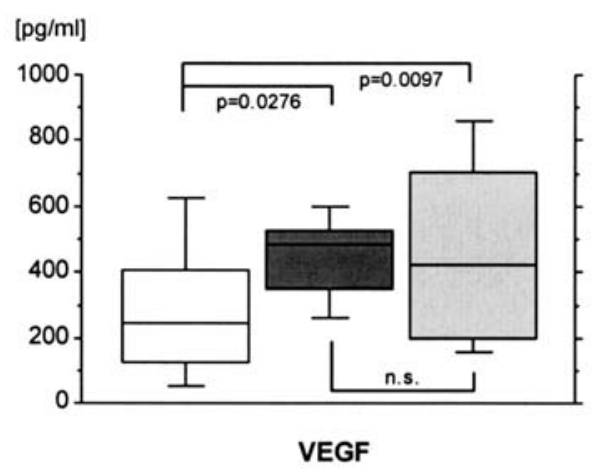

Progression of retinopathy - Prediction of retinal neovascularization. During the follow-up period, 10 of $32(30 \%)$ diabetic patients showed progression of retinopathy and developed either pre-proliferative or proliferative lesions. No significant differences for age, diabetes duration, prevalence of hypertension, and $\mathrm{HbA}_{1 \mathrm{c}}$ values between the subgroups with and without progression could be found. All progressors showed an ETDRS stage greater than stage 47B, six showed an ETDRS stage greater than 53E. Of the diabetic patients, 22 showed no progression and remained below ETDRS stage 35. VEGF and PEDF values are shown in the subgroups with and without a progression of retinopathy (Fig. 2B). Mean value of VEGF was $447 \mathrm{pg} / \mathrm{ml}$ in patients with retinopathy and was not different compared to patients without progression. However, PEDF content was found to be lower in patients with progression when compared to

Fig. 2A, B. VEGF-, PEDF-values in aqueous humor. (A) baseline values of VEGF and PEDF in control subjects and diabetic patients. (B) baseline content of VEGF and PEDF values in control subjects and the subgroups of diabetic probands, i.e. patients with and without progression of diabetic retinopathy
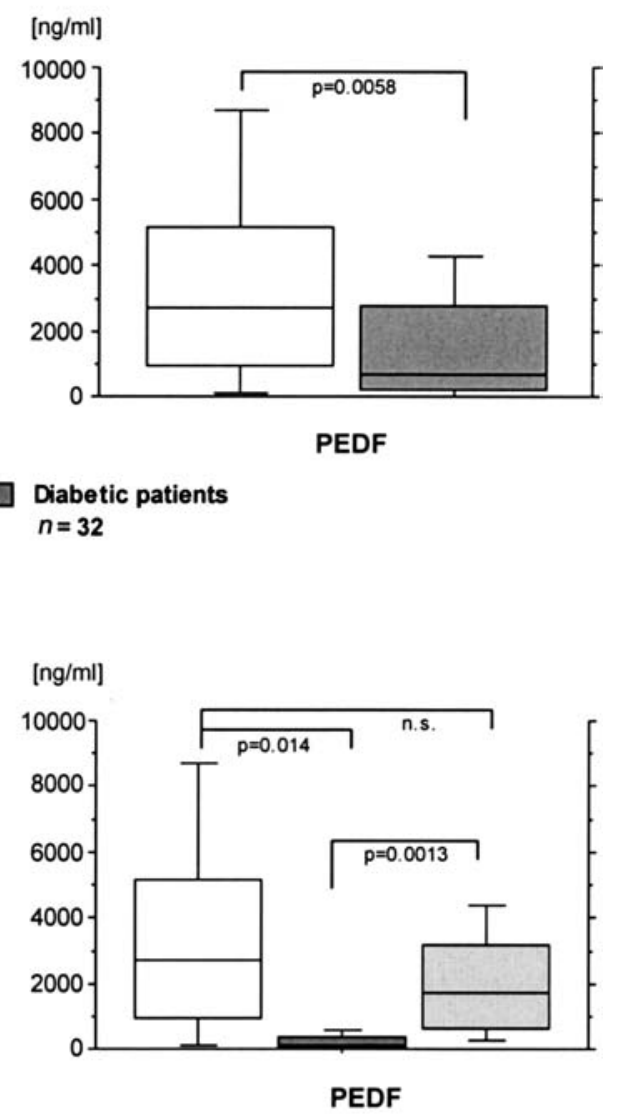


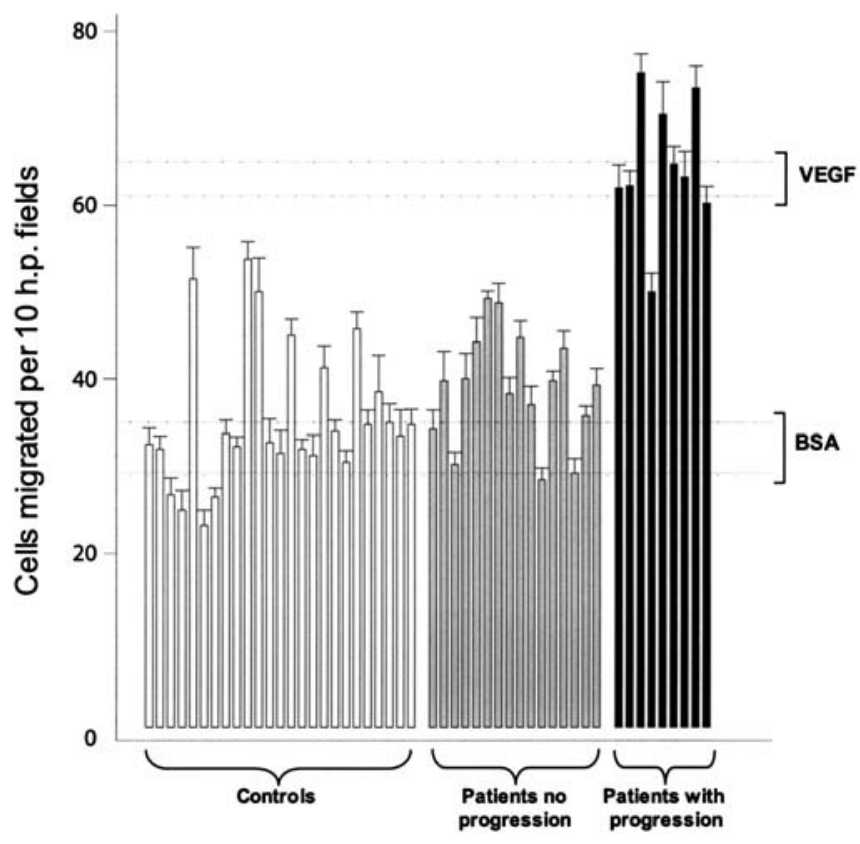

Fig. 3. Angiogenic activity as determined in modified Boyden chamber assay. Overall angiogenic activity determined by induction of chemotaxis of human dermal microvascular endothelial cells. No difference was seen between control subjects and diabetic patients without progression to advanced stages of retinopathy. Angiogenic activity was increased in the aqueous humor of patients who progressed to retinopathy (progressors vs. controls: $p=0.00005$; progressors - non-progressors: $p=0.0003$ ).

both control subjects $(p=0.014)$ and diabetic patients without progression $(p=0.013)$.

In vitro angiogenesis assay. Endothelial cell migration assays were carried out to determine angiogenic activity as a marker of angiogenesis. Activity was reflected by the ability of the samples to induce chemotaxis of neonatal human dermal microvascular endothelial cells. VEGF, PEDF-values, and migratory activity are shown in progressors and non-progressors compared to control subjects (Fig. 3). Progressors showed an increased angiogenic activity compared to non-progressors as well as control subjects.

The mean number of cells migrating towards the samples from non-diabetic patients was $35.51( \pm$ SD $8.01)$, and it was slightly higher (38.9 \pm SD 6.33$)$ in diabetic patients without progression of retinopathy. However, the migratory response in the aqueous humor of the subgroup of diabetic patients that progressed to advanced stages or retinopathy in the following years was almost doubled, with endothelial cell migration rising to $64.63( \pm$ SD 7.69$)$. This difference was highly significant when compared to control subjects $(p=0.0005)$, and when compared to the diabetic cohort without progression ( $p=0.003$; Fig. 3 ).

PEDF controls angiogenic activity in aqueous humor. To determine, if PEDF controls the activity of angio-

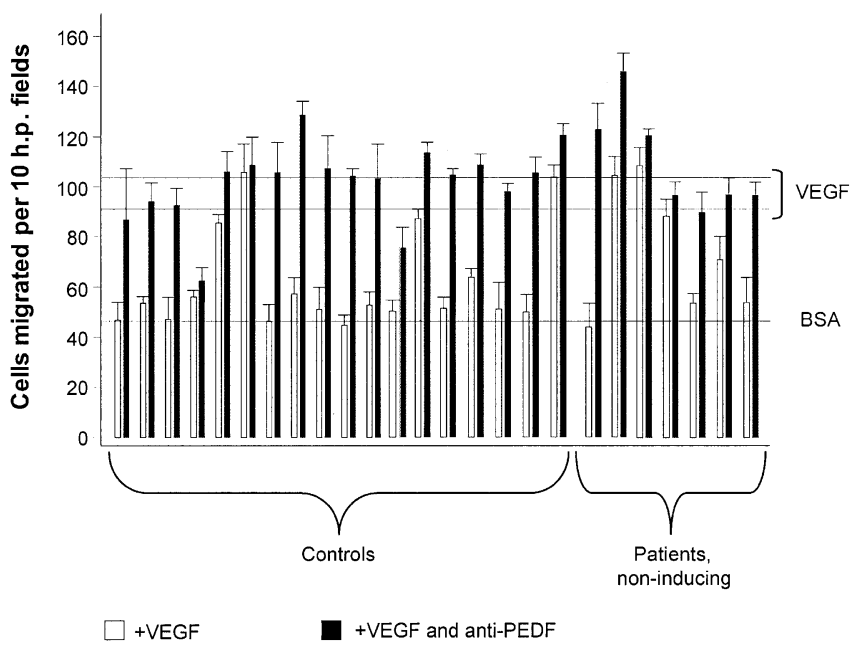

Fig. 4. Endothelial cell migration assay: The aqueous humor of most non-diabetic patients was anti-angiogenic due to PEDF. Samples that showed no angiogenic activity when tested alone, were re-tested for the ability to block VEGF induced migration of capillary endothelial cells (clear bars). The same samples were tested with VEGF combined with antibodies that neutralized PEDF (shaded bars), to see if inhibition was relieved when PEDF was inactivated. $200 \mathrm{pg} / \mathrm{ml}$ of VEGF was added to stimulate migration maximally

genic stimuli in the aqueous humor of the eye, the samples from probands that had not been angiogenic when tested alone were further examined in the in vitro assay for the ability to block migration of endothelial cells towards exogenously added VEGF. Relevant controls showed that the blocking anti-PEDF antibodies used at $20 \mu \mathrm{g} / \mathrm{ml}$ had no effect on basal or VEGF induced migration. Also purified PEDF at $500 \mathrm{ng} / \mathrm{ml}(10 \mathrm{nmol})$ effectively blocked migration towards VEGF and this inhibition was relieved as expected by anti-PEDF antibodies. The amount of VEGF added $(200 \mathrm{pg} / \mathrm{ml})$ is known to be able to stimulate vascular endothelial cells in vitro maximally. The majority of these samples turned out to be antiangiogenic in that they were capable of inhibiting endothelial cell migration up a gradient of VEGF (Fig. 4). In each cohort were some samples that were not inhibitory and the proportion of these was slightly greater in the diabetic non-inducing samples, but this difference was not significant.

This inhibitory effect was due to PEDF as it was lost upon addition of an affinity-purified antibody known to neutralize anti-angiogenic activity of PEDF (Fig. 4). This reversal of inhibition caused by removal of the angiogenic inhibitor PEDF was significant compared to baseline activity both in the non-diabetic cohort as well as in the diabetic cohort $(p=0.001$; $p=0.01$, respectively).

Predictive value of parameters tested. A logistic regression analysis was carried out to verify the association between angiogenic stimulators and angiogenic 
inhibitors as predictive markers for the progression of retinopathy. All variables used were defined at baseline; age, $\mathrm{HbA}_{1 \mathrm{c}}$, with or without hypertension, as well as diabetes duration and were not associated with progression. The only variables that could be identified as predictive were the content of PEDF ( $p=0.03)$, as well as in vitro angiogenic activity $(p=0.024)$ and the ratio of VEGF compared with PEDF ( $p=0.043)$.

\section{Discussion}

This study has addressed the roles of the angiogenic stimulator VEGF and the strong negative regulatory factor PEDF in the early stages of diabetic retinopathy in man. The analysis of samples from diabetic patients with no signs of advanced stages of diabetic retinopathy at baseline enabled us to determine pre-disease values of these mediators. By following the probands for almost 75 months it was found that PEDF-content was of predictive value for a significant progression of retinopathy. By use of the Boyden test we showed that angiogenesis was directly regulated by the PEDF content of the sample. A low content of the endogenous inhibitor PEDF in the aqueous humor predicted progression, whereas VEGF content was of no predictive value. The lack of association with classical risk factors for progression (i.e. $\mathrm{HbA}_{1 \mathrm{c}}$, hypertension, and diabetes duration) observed in our study might have been due to the selection of probands and due to the number of subjects recruited.

As expected from the studies of others analyzing vitreous fluids obtained during active proliferative disease $[9,10]$, we observed that aqueous humor in the diabetic state, even prior to the onset of retinopathy according to standard clinical criteria, is characterized by an increase of the physiologically relevant angiogenic stimulator VEGF. Yet VEGF content in aqueous humor could not predict progression to pathological neovascularization, nor were VEGF values related to the ability to induce migration. These data suggest that a rise in stimulatory factors in the early stages of diabetic retinopathy by itself might not be sufficient to induce the angiogenic switch thought to lead to progression of diabetic retinopathy $[13,14]$. It seems that a reciprocal fall in inhibitory PEDF is also needed. It seems likely that PEDF acts as a molecular brake on VEGF and other angiogenic stimuli and it is only when this brake fails that pathological retinopathy can develop $[15,16,20]$.

Our in vitro data show that, like the vitreous, the aqueous humor from normal individuals is typically anti-angiogenic due to PEDF $[15,20]$. This inhibitory activity is maintained in the majority of diabetic patients prior to onset of retinopathy but is lost in patients that have progressed significantly [20, 28, 29, $30,31]$. Recently an unbalanced expression of VEGF and PEDF in ischaemia-induced retinal neovascular- izations was shown [20]. Most importantly, this showed that systemic treatment with purified PEDF protein can completely ameliorate ischaemic retinopathy in a mouse model of retinopathy of prematurity [33]. Such efficacy suggests that PEDF or molecules mimicking its effect could provide a new way of treatment for a wide variety of diseases of the eye that involve pathological angiogenesis, vascular remodelling and vascular leakiness. The regulatory function of PEDF was recently found to be due to the specific dependence of remodelling vessels on Fas/Fas ligandmediated apoptosis [34].

Here we have provided clinical evidence that the concentration of the natural anti-angiogenic protein PEDF was of predictive value in the context of progression of diabetic retinopathy. A low value of PEDF predicted the development of the clinical signs of either pre-proliferative or proliferative retinopathy. PEDF, mimics of PEDF or other regulatory molecules of neo-angiogenesis could also be of considerable interest as novel treatment modalities in order to inhibit ocular neovascularization and delay progression to retinopathy $[20,33,36,37,38,39]$.

Acknowledgements. The help of Dr B Feldmann and Dr Anawars in patient recruitment is greatly acknowledged. We are indebted to Dr Schmid and Dr Fußgaenger for helpful discussions about the paper. Work was supported by grants from the Land BadenWuerttemberg to $\mathrm{BOB}$ and GL, and from Deutsche Forschungsgemeinschaft (SFB 518 to BOB), and from the American Heart Association (AHA SDG $0030023 \mathrm{~N}$ to OV), and NIH National Cancer Institute (CA64239 to NB). This work is dedicated to Dr. M. Pfeiffer and the late Prof. E.F. Pfeiffer for their establishment of a collaboration between ophtalmologists and endocrinologists in the treatment of diabetic eye disease at Ulm University.

\section{References}

1. Klein R (1997) The epidemiology of eye disease. In: Pickup JC, Williams G, (eds) Textbook of Diabetes, 2nd ed, Blackwell Sciences, Oxford 44:1-44.9

2. The Diabetes Control and Complications Trial Research Group (1993) The effects of intensive treatment of diabetes on the development and progression of long-term complications in insulin-dependent diabetes mellitus. $\mathrm{N}$ Engl J Med 329:977-986

3. The Diabetes Control and Complications Trial Research Group (2000) Retinopathy and nephropathy in patients with type 1 diabetes four years after a trial of intensive therapy. The Diabetes Control and Complications Trial/ Epidemiology of Diabetes Interventions and Complications Research Group. N Engl J Med 342:381-389

4. UK Prospective Diabetes Study (UKPDS) Group (1998) Intensive blood-glucose control with sulphonylureas or insulin compared with conventional treatment and risk of complications in patients with type 2 diabetes (UKPDS 33). Lancet 352:837-853

5. Keen H, Lee ET, Russell D, Miki E, Bennett PH, Lu M and the WHO Multinational Study Group (2001) The appearance of retinopathy and progression to proliferative reti- 
nopathy: the WHO multinational study of vascular disease in diabetes. Diabetologia 44: S22-S30

6. Kohner EM (1993) Diabetic retinopathy. BMJ 307:1195-1199

7. Porta M (1996) Endothelium: the main actor in the remodelling of the retinal microvasculature in diabetes. Diabetologia 39:739-744

8. Linsenmeier RA, Braun RD, McRipley MAet al. (1998) Retinal hypoxia in long-term diabetic cats. Invest Ophthalmol Vis Sci 39:1647-1657

9. Adamis AP, Miller JW, Bdernal M-Tet al.(1994) Increased vascular endothelial growth factor levels in the vitreous of eyes with proliferative diabetic retinopathy. Am J Ophthalmol 118:445-450

10. Aiello LP, Avery RL, Arrigg PG et al. (1994) Vascular endothelial growth factor in ocular fluid of patients with diabetic retinopathy and other retinal disorders. $\mathrm{N}$ Engl J Med 331:1480-1487

11. Yoshida A, Yoshida S, Khalil AK, Ishibashi T, Inomata $H$ (1998) Role of NF-kappaB-mediated interleukin-8 expression in intraocular neovascularization. Invest Ophthalmol Vis Sci 39:1097-1106

12. Aiello LP, Pierce EA, Foley ED et al. (1995) Suppression of retinal neovascularization in vivo by inhibition of vascular endothelial growth factor (VEGF) using soluble VEGFreceptor chimeric proteins. Proc Natl Acad Sci USA 92:10457-10461

13. Bouck N, Stellmach V, Hsu S (1996) How tumors become angiogenic. Adv Cancer Res 69:135-174

14. Hanahan D, Folkman J (1996) Patterns and emerging mechanisms of the angiogenic switch during tumorigenesis. Cell 86:353-364

15. Dawson DW, Volpert OV, Gillis P et al. (1999) Pigment epithelium-derived factor: a potent inhibitor of angiogenesis. Science 285:245-248

16. Chader GJ (2001) PEDF: raising both hopes and questions in controlling angiogenesis. Proc Natl Acad Sci USA 98:2122-2124

17. Becerra SP (1997) Structure-function studies on PEDF a noninhibitory serpin with neurotrophic activity. Adv Exp Med Biol 425:223-237

18. Miller AD, Robinson GS, Smith LEH, Becerra SP (2000) Regulation of pigment epithelium-derived factor (PEDF) production by oxygen. Invest Ophthalmol Vis Sci 41 [Suppl]: S869-S874

19. Gao G, Li Y, Zhang D, Gee S, Crosson G, Ma J (2001) Unbalanced expression of VEGF and PEDF in ischemia-induced retinal neovascularization. FEBS Lett 489:270-276

20. Bouck N (2002) PEDF: anti-angiogenic guardian of ocular function. Trends Mol Med 8:330-334

21. Cao W, Tombran-Tink J, Elias R, Sezate S, Mrazek D, McGinns JF (2001) In vivo protection of photoreceptors from light damage by pigment epithelium-derived factor. Invest Ophthalmol Vis Sci 42:1646-1652

22. Cayouette M, Smith SB, Becerra SP, Gravel C (1999) Pigment epithelium-derived factor delays the death of photoreceptors in mouse models of inherited retinal degenerations. Neurobiol Dis 6:523-532

23. Holekamp NM, Bouck N, Volpert O (2002) Pigment epithelium-derived factor is deficient in the vitreous of patients with choroidal neovascularization due to age-related macular degeneration. Am J Ophthalmol 134:220-227
24. Porta M, Sjoelie A-K, Chaturvedi N et al. (2001) Risk factors for progression to proliferative diabetic retinopathy in the EURODIAB Prospective Complications Study. Diabetologia 44:2203-2209

25. Anonymous (1998) Early worsening of diabetic retinopathy in the Diabetes Control and Complications Trial. Arch Ophthalmol 116:874-886

26. Chantelau E, Kohner EM (1997) Why some cases of retinopathy worsen when diabetic control improves. BMJ 315:1105-1106

27. Polverini PJ, Bouck NP, Rastinejad F (1991) Assay and purification of naturally occurring inhibitor of angiogenesis. Methods Enzymol 198:440-450

28. Spranger J, Osterhoff M, Reimann M et al. (2001) Loss of the antiangiogenic pigment epithelium-derived factor in patients with angiogenic eye disease. Diabetes 50:26412645

29. Okamoto T, Oikawa S, Toyota T (1990) Absence of angiogenesis-inhibitory activity in aqueous humor of diabetic rabbits. Diabetes 39:12-16

30. Gao G, Li Y, Zhang D, Gee S, Crosson G, Ma J (2001) Unbalanced expression of VEGF and PEDF in ischemia-induced retinal neovascularization. FEBS Lett 489:270-276

31. Okamoto T, Oikawa S, Toyota T (1990) Absence of angiogenesis-inhibitory activity in aqueous humor of diabetic rabbits. Diabetes 39:12-16

32. Stellmach V, Crawford SE, Zhou W, Bouck N (2001) Prevention of ischemia induced retinopathy by the natural ocular anti-angiogenic agent pigment epithelium-derived factor. Proc Natl Acad Sci USA 98:2593-2597

33. Volpert OV, Zaichuk T, Zhou W et al. (2002) Inducer-stimulated Fas targets activated endothelium for destruction by anti-angiogenic thrombospondin-1 and pigment epithelium-derived factor. Nature Med 8:349-357

34. Cayouette M, Smith SB, Becerra SP, Gravel C (1999) Pigment epithelium-derived factor delays the death of photoreceptors in mouse models of inherited retinal degenerations. Neurobiol Dis 6:523-532

35. Bilak MM, Corse AM, Bilak SR, Lehar M, Tombran-Tink J, Kuncl RW (1999) Pigment epithelium-derived factor (PEDF) protects motor neurons from chronic glutamatemediated neurodegeneration. J Neuropathol Exp Neurol 58:719-728

36. Cao W, Tombran-Tink J, Elias R, Sezate S, Mrazek D, McGinns JF (2001) In vivo protection of photoreceptors from light damage by pigment epithelium-derived factor. Invest Ophthalmol Vis Sci 42:1646-1652

37. Boehm BO, Lang GK, Jehle PM, Feldmann B, Lang GE (2001) Octreotide reduces risk for vitreous hemorrhages and loss of visual acuity in patients with high risk proliferative diabetic retinopathy. Horm Metab Res 33:300-306

38. Spranger J, Hammes H-P, Preissner KT, Schatz H, Pfeiffer AFH (2000) Release of the angiogenesis inhibitor angiostatin in patients with proliferative diabetic retinopathy: association with retinal photocoagulation. Diabetologia 43: 1404-1407

39. Mori K, Gehlbach P, Ando A, McVey D, Wei L, Campochiaro PA (2002) Regression of ocular neovascularization in response to increased expression of pigment epithelium-derived factor. Invest Ophthalmol Vis Sci 43:2428-2434 